\title{
Intelligent knowledge management in operations research
}

\author{
Gang Kou ${ }^{1} \cdot$ Lean $\mathbf{Y u}^{2}$
}

Published online: 28 August 2015

(c) Springer Science+Business Media New York 2015

Scientists employ sophisticated programs to mine and extract interesting information and knowledge from enormous databases. Intelligent knowledge, extracted from large data sets, reveals useful insights and hidden patterns. Intelligent knowledge management investigates the theoretical framework of data science and intelligent knowledge to bridge the gap between mining and knowledge management and further develops the techniques of how to identify and obtain the intelligent knowledge for decision-making.

Operations research (OR) and management science (MS) have strong influences on the development of intelligent knowledge management. Many OR methodologies and tools have been applied to knowledge sharing, knowledge organization, knowledge acquisition, and knowledge usage. It is important to synthesize the research findings of intelligent knowledge and OR with a wide range of real-life applications to support sustainable economic development.

This special volume includes both high-quality academic (theoretical or empirical) and practical papers that present current state-of-the-art results from researchers and practitioners from all related disciplines. All papers have some numerical or experimental illustrations of scientific value.

The guest editors hope that the papers published in this special volume will be of interest to academic and industrial communities by promoting high quality, novel and daring research findings. The volume provides solutions and tools to solve challenging problems faced by fellow researchers, practitioners, and students, as well as research challenges and initiatives for further research.

Gang Kou

kougang@yahoo.com

Lean $\mathrm{Yu}$

yulean@mail.buct.edu.cn

1 School of Business Administration, Southwestern University of Finance and Economics, Chengdu 611130, China

2 School of Economics and Management, Beijing University of Chemical Technology, Beijing 100029, China 
Acknowledgments The guest editors thank Professor Endre Boros, the Editor-in-Chief of the Annals of Operations Research for giving us the opportunity to edit this special volume. We thank all reviewers for their time, energy, and efforts to review the submissions. Last but not least, the guest editors thank the authors of all the submissions to this special volume for their contributions. We do appreciate having had the opportunity to supervise the reviewing process of the submissions. Without the support of all of you, it would have been impossible to compile this special volume for our readers. The guest editors acknowledge the grant support from the National Natural Science Foundation of China (\#71222108) and the National Science Fund for Distinguished Young Scholars (\#71025005). 